\title{
Exploring active galaxies with integral field spectroscopy
}

\author{
James E. H. Turner ${ }^{1}$, Bryan W. Miller ${ }^{1}$, \\ Joris Gerssen ${ }^{2}$ and Jeremy R. Allington-Smith ${ }^{2}$ \\ ${ }^{1}$ Gemini Observatory, Casilla 603, La Serena, Chile email: jturner@gemini.edu \\ ${ }^{2}$ University of Durham / Euro3D Research Training Network, \\ Department of Physics, University of Durham Science Laboratories, \\ South Road, Durham DH1 3LE, United Kingdom
}

\begin{abstract}
Integral Field Spectroscopy provides a powerful new tool for disentangling the complex structure of Active Galactic Nuclei-allowing 2D mapping of the distribution, kinematics and excitation of ionized gas and of stellar velocity profiles and populations. Such comprehensive datasets are likely to reveal important clues about the physics of the narrow line region, interactions with the host galaxy and central dynamical forces. Here we present observations of the central regions of NGC1068, obtained using the visible-wavelength GMOS-IFU at Gemini North and NGC4151, taken with a prototype near-infrared fibre IFU at the UK Infrared Telescope.
\end{abstract}

\section{Acknowledgements}

Based on observations obtained at the Gemini Observatory, which is operated by the Association of Universities for Research in Astronomy, Inc., under a cooperative agreement with the NSF on behalf of the Gemini partnership: the National Science Foundation (United States), the Particle Physics and Astronomy Research Council (United Kingdom), the National Research Council (Canada), CONICYT (Chile), the Australian Research Council (Australia), CNPq (Brazil), and CONICET (Argentina). The United Kingdom Infrared Telescope is operated by the Joint Astronomy Centre on behalf of the U.K. Particle Physics and Astronomy Research Council.

\section{References}

Cappellari, M., \& Copin, Y. 2003, MNRAS, 342, 345

Cecil, G., Dopita, M. A., Groves, B., Wilson, A. S., Ferruit, P., Pécontal, E., \& Binette, L. 2002, ApJ, 568, 627

Haynes, R., Content, R., Turner. J. E. H., Allington-Smith, J. R., \& Lee, D. 1998, Proc. SPIE. 3354,419

Haynes, R., et al. 1999, PASP, 111, 1451

Kaiser, M. E., et al. 2000, ApJ, 528, 260

Turner, J. E. H., et al. 2002, MNRAS, 331, 284 

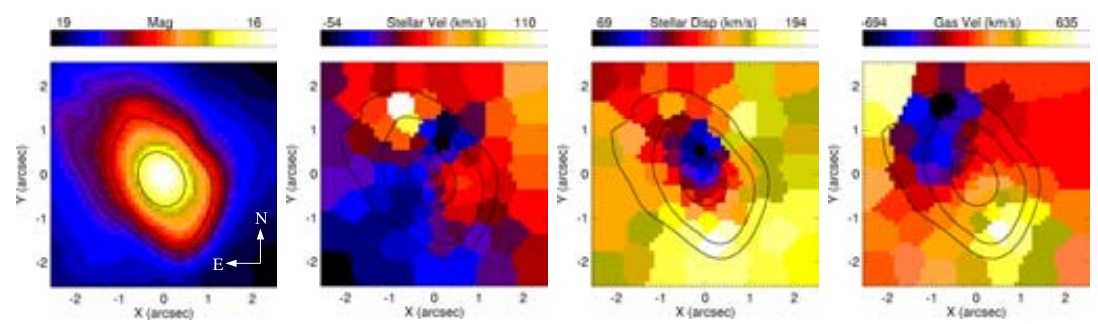

Figure 1. An image of the central 5" of NGC 1068 (first panel) was obtained by summing a datacube from the IFU observations in wavelength between $5100-5400 \AA$. The preliminary analysis in the next 3 panels uses data that were co-added into spatial bins of $>=75$ using a Voronoi tessellation scheme (Cappellari \& Copin 2003). The stellar velocity field (second panel) and the stellar velocity dispersion (third panel) were derived by fitting a template spectrum (type K0III), convolved with a Gaussian kernel, to the spectral region around the Mgb triplet $(\sim 5170 \AA)$. The large central dip in velocity dispersion is the result of poor fitting where the spectrum is dominated by non-continuum emission. The final panel shows the velocity field of (a subset of) the gas, derived by fitting single Gaussian profiles to the [O III] and $\mathrm{H} \beta$ emission lines. Note that the gas velocity field exhibits symmetry perpendicular to the stellar rotation.
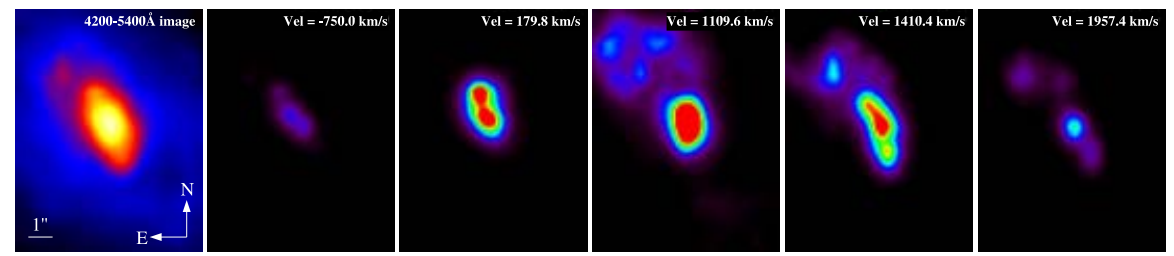

Figure 2. [O III] emission from gas at different velocities. The left-hand panel is a reconstructed $4200-5400 \AA$ image of the central $8 \times 10$ " of NGC 1068. Subsequent panels show images in [O III] emission at different wavelengths, with the corresponding observed velocities printed in the upper right. The systemic velocity of the galaxy is $1137 \mathrm{~km} \mathrm{~s}^{-1}$. Cecil et al. (2002) interpret the redshifted emission to the NE as being due to the approaching radio lobe expanding into the galactic disc. We have been working on methods for mapping the $2 \mathrm{D}$ velocity field in multiple components, to give a more analytical picture of the kinematics.
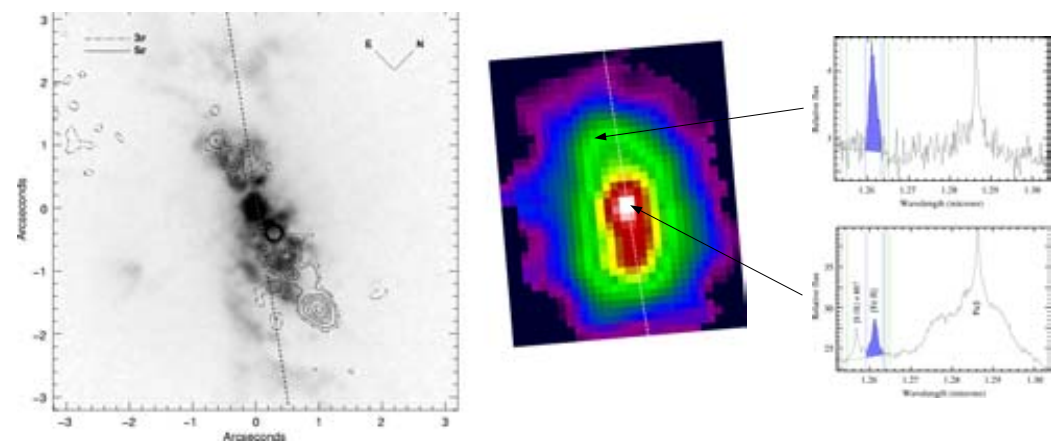

Figure 3. A comparison of the extended $1.257 \mu \mathrm{m}$ [Fe II] emission in NGC 4151 with the optical Narrow Line Region and the radio jet. The left-hand panel shows an HST image in [O III] emission overlaid with radio contours (from Kaiser et al. 2000). The central panel is a map of the [Fe II] line from the SMIRFS IFU data, shown on the same scale and orientation. Dotted lines mark the (average) axis of [Fe II] emission on both plots. The right-hand panel shows two example J-band spectra from different spatial points in the datacube, with annotation showing how the [Fe II] flux was measured. We find that orientation and shape of the [Fe II] map is similar to the NLR and less compatible with the direction of the jet, suggesting that the forbidden emission arises primarily through photoionization rather than shocking (Turner et al. 2002). 